\title{
Intrarenal urothelial cancers confused as infiltrative renal masses: Report of 22 cases and literature review
}

\author{
XIAOBO DING ${ }^{1}$, XIAOBO $\mathrm{MA}^{2}$, YATAO JIA ${ }^{3}, \mathrm{HONGFEI} \mathrm{LI}^{3}$ and YANBO WANG ${ }^{3}$ \\ Departments of ${ }^{1}$ Radiology, ${ }^{2}$ Pathology and ${ }^{3}$ Urology, First Hospital of Jilin University, Changchun, Jilin 130021, P.R. China
}

Received February 16, 2018; Accepted May 22, 2018

DOI: $10.3892 / \mathrm{ol} .2018 .8867$

\begin{abstract}
Distinguishing infiltrative renal masses (IRMs) from intrarenal urothelial cancers (IUCs) is critically important, but may be challenging for any radiologist or urologist. The present study aimed to summarize the clinical, imaging and pathological characteristics of IRM, which were postoperatively confirmed as IUC. The analysis was performed using the records of 22 patients who were preoperatively diagnosed with IRM but the results of percutaneous biopsies or postoperative pathological analyses led to diagnoses of urothelial cancers (UCs) from January 2011 to December 2017. The demographic data, computed tomography (CT) imaging features and pathological characteristics were evaluated. The present study also reviewed the literature concerning the IRM and IUC. The mean age of patients was 62 years and $86.4 \%$ of them were $>55$ years. The sex and tumor side distributions were equal. Hematuria and/or flank pain were observed in $86.4 \%$ of patients. All patients exhibited endophytic solid renal masses with unclear tumor boundaries on CT images. The kidneys of $81.8 \%$ of patients maintained their normal shape while mild alternations were observed in $18.2 \%$ of cases. A total of $81.8 \%$ of patients maintained the reniform shape and $18.2 \%$ exhibited mild contour change. Of all patients, all tumors exhibited less or equal attenuation on unenhanced CT images and they were mildlyimproved on enhanced CT. A total of 6 cases were confirmed by biopsy, when patients underwent laparoscopic nephroureterectomy instead of radical nephrectomy. The remaining 16 patients underwent laparoscopic nephrectomy but the postoperative pathological diagnoses revealed the presence of UCs. All postoperatively confirmed cancers were stages T3 and T4 (62.5 and 37.5\%, respectively). UCs should be suspected in middle aged or elderly middle-elderly patients presenting renal masses with endophytic solid unclear tumor boundary on unenhanced and slightly enhanced CT images,
\end{abstract}

Correspondence to: Dr Yanbo Wang, Department of Urology, First Hospital of Jilin University, 71 Xinmin Street, Changchun, Jilin 130021, P.R. China

E-mail: wangyanb@jlu.edu.cn

Key words: intrarenal urothelial cancers, infiltrative renal masses, case report, literature review accompanied with hematuria and/or flank pain. Preoperative biopsy is preferred for complicated cases.

\section{Introduction}

The most common type of malignant kidney cancer is renal cell carcinoma (RCC), which accounts for $85-90 \%$ of cases (1). Primary urothelial cancer (UC) of the upper tract is only diagnosed in about $7-10 \%$ of all patients with renal tumors $(2,3)$. Macroscopic hematuria, flank pain and palpable flank mass are three main clinical presentation of RCC but such typical tumors are rare at present due to increasing use of imaging (4). Microscopic or macroscopic hematuria is the most common symptom of UC and symptomatic hydronephrosis occurs if the tumor locates at the ureteropelvic junction (5). Both RCC and $\mathrm{UC}$ are more common in men vs. women. RCC originates from proximal convoluted tubule including clear cell renal carcinoma, papillary RCC, chromophobe RCC, collecting duct RCC, renal medullary carcinoma and sarcomatoid RCC (6). While UC arises from the transitional epithelium, including papillary and non-papillary transitional cell carcinomas. Nephron sparing surgery (NSS) or radical nephrectomy are the main procedures for RCC, and radical nephroureterectomy (RNU) with en bloc excision of the periureteric bladder cuff is the standard surgical treatment for UCs $(7,8)$. Computed tomography (CT) is mostly used to preoperative diagnosis of RCC and UC. Typically, the tumors of RCC are soft tissue attenuation and sometimes accompanied with necrosis and calcification, which have stronger or irregular enhancement during the corticomedullary phase. In contrast, for UC, filling defect or distortion of calyces are shown on CT scan and the tumors have mild enhancement during contrast CT $(9,10)$. However, unusual infiltrative renal masses (IRMs) are similar to intrarenal UCs (IUCs) on imaging, which frequently results in misdiagnosis and mistreatment. The present study retrospectively evaluated the patients who were preoperatively diagnosed with IRM, but the subsequent pathological results confirmed UCs in First Hospital of Jilin University. A comprehensive review of the literature was also performed.

\section{Case report}

The study protocol was approved by the Institutional Review Board of the First Hospital of Jilin University (Jilin, China). A total of 22 IRM patients with pathologically confirmed IUCs 
A

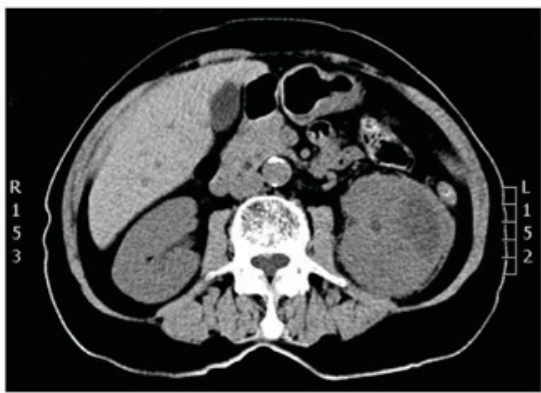

C

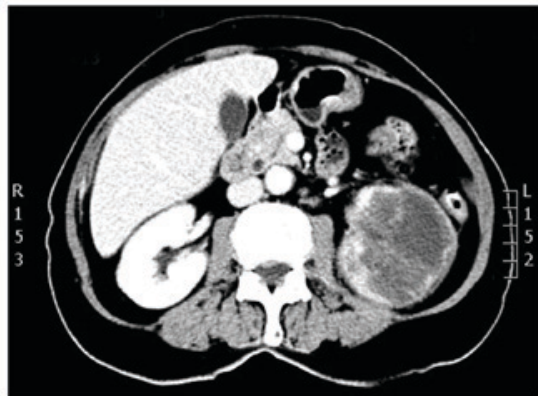

B

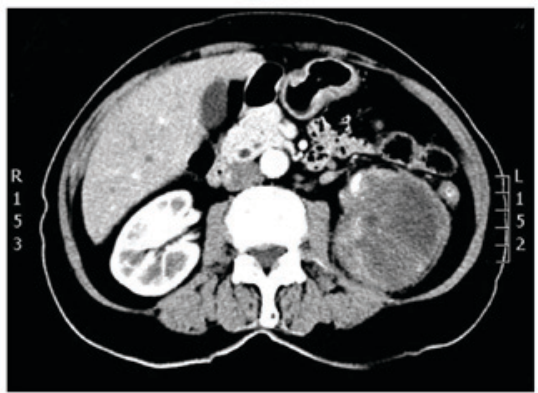

D

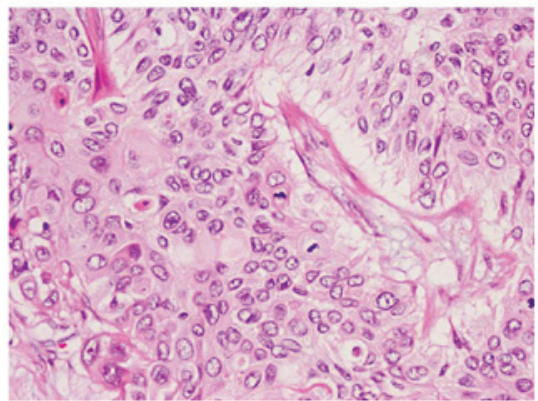

Figure 1. A 74-year-old female with a left renal mass. (A) A poorly defined isodense mass was shown on the axial unenhanced CT scan. Mild enhancement occurred on the (B) cortical and (C) nephrographic phases, showing an infiltrative, solid and unclear boundary. (D) Microscopic analysis demonstrated an infiltrative growth of tumor tissues, highly heterogeneous cells with irregular contours, abundant eosinophilic cytoplasm and vacuolar nuclei (hematoxylin and eosin staining, magnification, $\mathrm{x} 400)$. CT, computed tomography.
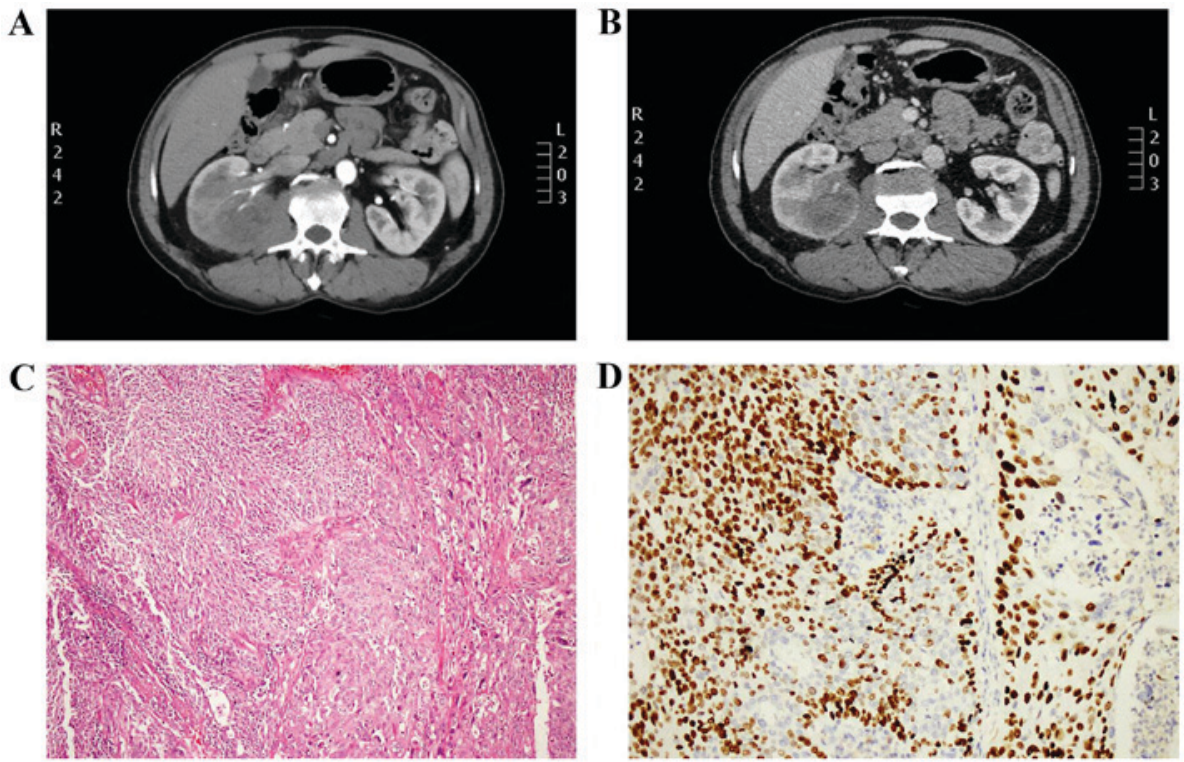

Figure 2. A 59-year-old male with a right renal mass. The contrast-enhanced CT imaging illustrated a mild enhancement on the (A) cortical and (B) nephrographic phases, exhibiting an endophytic, solid, preserved contour and an unclear boundary. (C) Microscopic analysis indicated papillary infiltrative growth pattern of tissue. Left was the typical UC and the right was squamous differentiation (hematoxylin and eosin staining, magnification, x100). (D) Positive expression of P63 in tumor cells (immunohistochemical staining, magnification, x200). CT, computed tomography; UC, urothelial cancer.

at our institution from January 2011 to December 2017 were included in the present study. Representative cases are shown in Figs. 1 and 2. Detailed clinical and pathological data was available for all patients and the CT images were all performed in First Hospital of Jilin University (Jilin, China).

Lists of demographic and pathological data of the study cohort are shown in Table I. There were 14 males and 8 females with a median age of 62 years. A total of 11 patients were between $56-65$ years $(50 \%)$ and 8 patients were $>65$ years old $(36.4 \%)$. There was no difference between the frequency of occurrence of tumors on the left or right side (11 vs. 10 cases, respectively). Hematuria and/or flank pain were the main symptoms which accounted for $86.4 \%$ of patients. One patient was diagnosed during a routine physical examination and another two were initially admitted due to emaciation. Two patients exhibited symptoms of lung metastasis. A total of six patients $(27.3 \%)$ who underwent laparoscopic nephroureterectomy were diagnosed by percutaneous biopsy. The remaining 16 patients underwent laparoscopic radical nephrectomy but the postoperative pathology led to diagnosis of UCs, among 
Table I. Demographic and pathological data on 22 patients.

\begin{tabular}{|c|c|}
\hline Parameters & No. of patients $(\%)$ \\
\hline \multicolumn{2}{|l|}{ Age, years ${ }^{\mathrm{a}}$} \\
\hline $45-55$ & $3(13.6)$ \\
\hline $56-65$ & $11(50)$ \\
\hline$>65$ & $8(36.4)$ \\
\hline \multicolumn{2}{|l|}{ Sex } \\
\hline Male & $14(63.6)$ \\
\hline Female & $8(36.4)$ \\
\hline \multicolumn{2}{|l|}{ Side } \\
\hline Left & $11(50)$ \\
\hline Right & $10(45.5)$ \\
\hline Both & $1(4.5)$ \\
\hline \multicolumn{2}{|l|}{ Symptoms } \\
\hline No & $1(4.5)$ \\
\hline Hematuria & $6(27.3)$ \\
\hline Flank pain & $6(27.3)$ \\
\hline Hematuria and flank pain & $7(31.8)$ \\
\hline Emaciation & $2(9)$ \\
\hline Suspected lung metastasis & $2(9)$ \\
\hline Biopsy & $6(27.3)$ \\
\hline \multicolumn{2}{|l|}{ Tumor stage } \\
\hline pT1 & $0(0)$ \\
\hline pT2 & $0(0)$ \\
\hline pT3 & $10(62.5)$ \\
\hline pT4 & $6(37.5)$ \\
\hline Positive lymph node & $2(9)$ \\
\hline
\end{tabular}

${ }^{a}$ The median age was 62 years (range, $45-80$ years).

which, 10 (62.5\%) were stage T3 and 6 (37.5\%) were stage T4. A total of two patients were confirmed positive lymph node.

All tumor features identified in the CT images were shown in Table II. The mean maximum diameter was $4.8 \mathrm{~cm}$. All tumors were endophytic and solid. All tumor boundaries were unclear. The reniform contours of the operated kidneys were completely preserved or mildly altered (81.8 and $18.2 \%$, respectively). In unenhanced CT images, all tumors exhibited isoattenuation or hypoattenuation which were mildly enhanced on enhanced CT images. A total of 2 patients demonstrated lymph node enlargement.

\section{Discussion}

The surgical treatment and postoperative management of kidney cancers and renal pelvis cancers are significantly different. Therefore, distinguishing IRMs from IUCs is critically important but sometimes challenging (11). UC of the renal pelvis may display an infiltrative growth pattern, which results in a close radiological mimic of IRM.

Certain studies have attempted to differentiate IRM from IUC but the limitations were obvious. Raza et al (12) reviewed CT studies of 64 centrally located RCC and 34 IUC and found that the presence of a tumor centered within the collecting
Table II. CT imaging features of tumor.

\begin{tabular}{|c|c|}
\hline Parameters & No. of patients $(\%)$ \\
\hline Mean maximus diameter, $\mathrm{cm}$ (range) & $4.83 .6-6.5$ \\
\hline \multicolumn{2}{|l|}{ Location } \\
\hline Endophytic & $22(100)$ \\
\hline Exophytic & $0(0)$ \\
\hline \multicolumn{2}{|l|}{ Reniform shape } \\
\hline No & $18(81.8)$ \\
\hline Mild & $4(18.2)$ \\
\hline Moderate & $0(0)$ \\
\hline Severe & $0(0)$ \\
\hline \multicolumn{2}{|l|}{ Tumor boundary } \\
\hline Clear & $0(0)$ \\
\hline Unclear & $22(100)$ \\
\hline \multicolumn{2}{|l|}{ Component } \\
\hline Cystic & $0(0)$ \\
\hline Necrosis & $0(0)$ \\
\hline Solid & $22(100)$ \\
\hline \multicolumn{2}{|l|}{ Unenhanced CT density } \\
\hline High attenuation & $0(0)$ \\
\hline Isoattenuation/hypoattenuation & $22(100)$ \\
\hline \multicolumn{2}{|l|}{ Degree of enhancement on CT } \\
\hline Mild & $22(100)$ \\
\hline Moderate & $0(0)$ \\
\hline Severe & $0(0)$ \\
\hline Lymph node enlargement & $2(9)$ \\
\hline
\end{tabular}

The mean maximus diameter was $4.8 \mathrm{~cm}$ (range, $36-6.5 \mathrm{~cm}$ ). CT, computed tomography.

system was the most valuable characteristic identified on the CT images. Bata et al (13), concluded that using multiple small ROIs was valuable for distinguishing IRM from IUC. However, based on one case and literature review, Li et al (14) reported that imaging results of hypovascular RCC were indistinguishable from IUC. Han et al (15), attempted to evaluate the potential systemic inflammatory markers to differentiate between infiltrative RCC and infiltrative UCs. Their analysis indicated that age and lymphocyte-monocyte ratio were significantly different between patients with IRM. To the best of our knowledge, the present study is the first to comprehensively evaluate clinical, imaging and pathological characteristics of IRMs that were postoperatively confirmed as IUCs.

In the present study, $63.6 \%$ of the patients were male and $36.4 \%$ were female. There was no obvious tendency about sex. Zhu et al (16) retrospectively assessed 29 patients with invasive renal parenchymal urothelial carcinoma (IRPUC) and found that IRPUC was more likely to occur in the right kidney $(82.7 \%)$. However, in the present study, the ratio of malignancies in left and right kidneys was 11 vs. 10. Raza et al (12), also supported our results. It was observed that prevalence of the disease was increased among middle-elderly patients, over 56 years old $(86.4 \%)$, which suggests that older age may be associated with the development of IRC. 
The mean maximum diameter of tumors was between 3.6 and $6.5 \mathrm{~cm}$. A previous study demonstrated that if such tumors were renal cancers, hematuria and flank pain were infrequent (17). However, in the present study, $27.3 \%$ of patients exhibited either a symptom of hematuria or flank pain and $31.8 \%$ exhibited both hematuria and flank pain, which indicated that hematuria and/or flank pain were the principal predictive symptoms.

The CT images revealed that all 22 tumors were endophytic and solid. All patients were free from cystic or necrotic alterations. The results were similar to those previously reported by Raza et al (12). RCC may be associated with a moderate or severe renal shape distortion, however, in the present study, a total of 18 patients $(81.2 \%)$ retained the renal contour and 4 patients $(18.2 \%)$ underwent mild alteration of shape. However, IRM does not necessarily alter the reniform contour in all patients and certain patients with IUC can sometimes exhibit a distorted renal shape $(18,19)$. An unclear tumor boundary was identified in each case included in the present study, which was an important and valuable CT characteristic. Isoattenuation or hypoattenuation occurred in all patients and were visible in unenhanced CT images and all tumors exhibited poor homogeneous enhancing masses in contrast-enhanced CT images.

Although, several special CT features can help distinguish IRM from IRC, in the majority of cases, other similar neoplasms are present, including renal lymphoma, collecting duct carcinoma, metastasis, xanthogranulomatous pyelonephritis and medullary carcinoma $(20,21)$. Biopsy is a necessary procedure in case of misdiagnosis and mistreatment. Guarnizo et al (22), assessed the diagnostic accuracy of ureteroscopic biopsy and found that ureteroscopic multi-biopsy can lead to an accurate diagnosis of urothelial carcinoma in $89 \%$ of cases and can predict the exact histopathological grade in $78 \%$ of cases. Furukawa et al (23), assessed a total of 40 patients and found that ureteroscopic biopsy could lead to determination of the pathological grade of nephroureterectomy specimens with an accuracy rate of $87.5 \%$. Recently, Huang et al (24) reviewed 26 cases of upper tract lesions and found that percutaneous biopsy was also a safe and effective method.

In conclusion, it is challenging to thoroughly make a distinction between IRM and IUC preoperatively; however, the elder accompanied with hematuria and/or flank pain are more likely to indicate IRM, who with special CT features: endophytic, solid and unclear tumor boundary on unenhanced CT and slightly enhancement on contrast-enhanced CT. Pre-operative endoscopic or percutaneous biopsy, is a valuable tool for complex cases.

\section{Acknowledgements}

Not applicable.

\section{Funding}

Dr. Wang was funded by the China Scholarship Council (grant no. 201706175045).

\section{Availability of data and materials}

The datasets used and/or analyzed during the current study are available from the corresponding author on reasonable request.

\section{Authors' contributions}

$\mathrm{XD}, \mathrm{XM}, \mathrm{YJ}$ and HL and YW were all involved in this conception of this study and helped to draft the manuscript. All authors have read and approved the final manuscript.

\section{Ethics approval and consent to participate}

Institutional Review Board of the First Hospital of Jilin University (Jilin, China). Verbal informed consent was obtained from the patients.

\section{Consent for publication}

Verbal informed consent was obtained from the patients.

\section{Competing interests}

The authors declare that they have no competing interests.

\section{References}

1. Sheir KZ, El-Azab M, Mosbah A, El-Baz M and Shaaban AA: Differentiation of renal cell carcinoma subtypes by multislice computerized tomography. J Urol 174: 451-455, 2005.

2. Mandalapu RS and Matin SF: Contemporary evaluation and management of upper tract urothelial cancer. Urology 94: 17-23, 2016.

3. Arancibia MF, Bolenz C, Michel MS, Keeley FX Jr and Alken P: The modern management of upper tract urothelial cancer: Surgical treatment. BJU Int 99: 978-981, 2007.

4. Rossi SH, Klatte T, Usher-Smith J and Stewart GD: Epidemiology and screening for renal cancer. World J Urol, 2 Apr 2018 (Epub ahead of print).

5. Jaworski D, Szylberg Ł, Gzil A, Stawinski P, Kasperska A and Marszałek A: Diagnostic difficulties in cases of papillary urothelial neoplasm of low malignant potential, urothelial proliferation of uncertain malignant potential, urothelial dysplasia and urothelial papilloma: A review of current literature. Ann Diagn Pathol, 17 Dec 2017 (Epub ahead of print).

6. Czarniecki M, Gautam R, Choyke PL and Turkbey B: Imaging findings of hereditary renal tumors, a review of what the radiologist should know. Eur J Radiol 101: 8-16, 2018.

7. Pierorazio PM, Johnson MH, Patel HD, Sozio SM, Sharma R, Iyoha E, Bass EB and Allaf ME: Management of renal masses and localized renal cancer: Systematic review and meta-analysis. J Urol 196: 989-999, 2016.

8. Soria F, Shariat SF, Lerner SP, Fritsche HM, Rink M, Kassouf W, Spiess PE, Lotan Y, Ye D, Fernández MI, et al: Epidemiology, diagnosis, preoperative evaluation and prognostic assessment of upper-tract urothelial carcinoma (UTUC). World J Urol 35: 379-387, 2017.

9. Nabi S, Kessler ER, Bernard B, Flaig TW and Lam ET: Renal cell carcinoma: A review of biology and pathophysiology. F1000Res 7: 307, 2018.

10. Froemming A, Potretzke T, Takahashi N and Kim B: Upper tract urothelial cancer. Eur J Radiol 98: 50-60, 2018.

11. Hartman DS, Davidson AJ, Davis CJ Jr and Goldman SM: Infiltrative renal lesions: CT-sonographic-pathologic correlation. AJR Am J Roentgenol 150: 1061-1064, 1988.

12. Raza SA, Sohaib SA, Sahdev A, Bharwani N, Heenan S, Verma H and Patel U: Centrally infiltrating renal masses on CT: differentiating intrarenal transitional cell carcinoma from centrally located renal cell carcinoma. AJR Am J Roentgenol 198: 846-853, 2012.

13. Bata P, Tarnoki DL, Tarnoki AD, Novak PK, Gyebnar J, Kekesi D, Szendroi A, Fejer B, Szasz AM, Nyirady P, et al: Transitional cell and clear cell renal carcinoma: Differentiation of distinct histological types with multiphase CT. Acta Radiol 55: 1112-1119, 2014.

14. Li Y, Ding YU, Chen D, Yu Z, Gui Y, Yang S and Lai Y: Renal cell carcinoma growing into the renal pelvis and mimicking transitional cell carcinoma: A case report and literature review. Oncol Lett 9: 1869-1872, 2015. 
15. Han JH, Yoon YE, Kim SY, Cho YI, Rha KH, Choi YD and Han WK: Preoperative lymphocyte-monocyte ratio ameliorates the accuracy of differential diagnosis in non-metastatic infiltrative renal masses. Yonsei Med J 58: 388-394, 2017.

16. Zhu Q, Zhu W, Wu J and Chen W: Multidetector CT imaging features of invasive renal parenchyma urothelial carcinoma. Br J Radiol 89: 20151068, 2016.

17. Ginzburg S, Tomaszewski JJ and Kutikov A: Focal ablation therapy for renal cancer in the era of active surveillance and minimally invasive partial nephrectomy. Nat Rev Urol 14: 669-682, 2017.

18. Prando A, Prando P and Prando D: Urothelial cancer of the renal pelvicaliceal system: Unusual imaging manifestations. Radiographics 30: 1553-1566, 2010.

19. Yoon SK, Nam KJ, Rha SH, Kim JK, Cho KS, Kim B, Kim KH and Kim KA: Collecting duct carcinoma of the kidney: CT and pathologic correlation. Eur J Radiol 57: 453-460, 2006.
20. Blitman NM, Berkenblit RG, Rozenblit AM and Levin TL: Renal medullary carcinoma: CT and MRI features. AJR Am J Roentgenol 185: 268-272, 2005.

21. Yan Y, Liu L, Zhou J, Li L, Li Y, Chen M, Wang L, He W, Guan X, Zu X and Qi L: Clinicopathologic characteristics and prognostic factors of sarcomatoid renal cell carcinoma. J Cancer Res Clin Oncol 141: 345-352, 2015.

22. Guarnizo E,Pavlovich CP, Seiba M, Carlson DL, Vaughan ED Jr and Sosa RE: Ureteroscopic biopsy of upper tract urothelial carcinoma: Improved diagnostic accuracy and histopathological considerations using a multi-biopsy approach. J Urol 163: 52-55, 2000.

23. Furukawa J, Miyake H, Sakai I and Fujisawa M: Significance of ureteroscopic biopsy grade in patients with upper tract urothelial carcinoma. Curr Urol 6: 156-159, 2013.

24. Huang SY, AhrarK, Gupta S, Wallace MJ,Ensor JE, Krishnamurthy S and Matin SF: Safety and diagnostic accuracy of percutaneous biopsy in upper tract urothelial carcinoma. BJU Int 115: 625-632, 2015. 